\title{
ESOVYM - Enermeter's system of vineyard monitoring
}

\author{
Manuel João Ferreira ${ }^{1}$, André Pinho ${ }^{1}$, António Graça ${ }^{2}$, and Teresa Martins ${ }^{1}$ \\ ${ }^{1}$ ENERMETER, Computer Vision Department, Celeirós, Braga, Portugal \\ ${ }^{2}$ Sogrape Vinhos S.A., Research and Development Department, Aldeia Nova, Avintes, Portugal
}

\begin{abstract}
This paper presents the results of the national project QREN number 24792 that consisted on the development of a mobile unit for acquiring and processing georeferenced images, to be applied in viticulture. The system has high resolution (approximately $2 \mathrm{~cm}^{2}$ ) and moves through the entire surface of the vineyard and of the field portions. During displacement, this unit performs simultaneously the acquisition of images (in the visible and infrared spectrum) of left and right trellis for processing, analysis and visualization. The mobile unit is not autonomous, which means that it is linked to a $4 \times 4$ moto or to any agriculture tractor. It is easy to operate and has low cost, which allows its use at different stages of vineyard production.
\end{abstract}

\section{Introduction}

The high spatial and temporal variability of an agriculture exploration with specific demands, such as a vineyard, represents the opportunity to use variable rate technologies, such as the methodologies of precision viticulture. Among technologies used to obtain maps from vigour and vegetative state of plants, remote detection is one of the most common. This type of technology is based on computer vision techniques and typically combines the use of red and infrared bands to create indexes of vigour, vegetative state or others. Although widely used, remote detection has several important limitations. For instance, the need to validate in the field maps obtained from remote detection since usually images communicate only information from the top and not from the entire canopy. In addition, depending on the resolution and time of the day, among other factors, information carried by the image may represent the behaviour of the vineyard and also of the vegetation between trellises.

With the advance of integrated technologies based on robotics, solutions supported by UAVs started to appear in the market. However, the operators of these systems must have a high degree of specialization as they need to command the UAV and they have to develop tasks with different softwares, responsible for specific parts of the solution.

The system presented in this work, developed by ENERMETER during the Portuguese project QREN number 24792, is supported by computer vision and georeferenced sensors. The resulting system allows for acquisition of $100 \%$ of the target area of the vineyard and of entire vines' canopies, generating high resolution maps of the whole leaf surface without need for expertise in navigation or software. The operator can access all acquired images and, so, he may perform remote validation of generated maps.

\section{Remote detection - state of the art}

To ensure proper management of human and equipment resources in vineyard production and, consequently, for high quality wine production, it is essential to efficiently manage at different phenological stages of the growth cycle. Those are highly related with vine growth and with quality of the grape.

Remote detection is one of the techniques used to help predicting vineyard growth, through the use of aerial images obtained from multispectral sensors, typically acquired from RGB and from infrared (IR) cameras [1]. Image acquisition can be done through the use of satellite images or through images acquired by airplanes. In last years, acquisition by airplanes has been replaced or complemented with Unmanned Aerial Vehicles (UAV).

Satellite images have low spatial and temporal resolutions and high cost. On the contrary, remote detection performed from aerial platforms has higher resolution, both spatial and temporal. The former can be from a few centimeters to one meter [3-7]. Using an aerial vehicle, temporal resolution is basically dependent on needs and weather conditions.

With the advance of robotics, specifically of mobile robots, guidance and automatic trajectories generation, new methods for acquiring remote images have appeared, specifically those based on UAVs. Some of these UAVs have coupled the necessary technology to perform remote detection: visible and infrared cameras and GPS. These units can obtain images with even higher resolution than aerial vehicles but at low cost.

From remote detection images it is possible to quantify a set of indexes that can be used to predict the vigour of the vine, such as $[8,4,9]$ :

- NDVI - "Normalised Difference Vegetation Index";

- PCD - "Plant Cell Density";

- RVI - "Ratio Vegetation Index. 
From these indicators it is possible to identify areas inside the vineyard needing different intervention procedures during the vine growth, making use of variable rate methodology [3].

In the literature, there are several works $[10,11]$ that seek correlation between indicators obtained by remote sensing images and productive factors, such as leaf area and production of grapevines. High correlations allow to predict the outcome of production and to implement better management of surrounding resources.

\section{System architecture}

The system architecture must be able to face the actual limitations of remote detection techniques, which are the following:

- information that is delivered to the vineyard manager is limited to the top of the canopy, which can be confused with soil, depending on the resolution of the system and on the soil cover;

- indexes may suggest the existence of stress on the vine, but using only the aerial image is not possible to identify which type of stress; it is necessary to go to vineyards for further analysis;

- indicators obtained by aerial images may not reflect the true state of the vineyard in situations of very dense or sparse density of leaf area;

- from currently used indicators it is difficult to infer (low correlations), with accuracy, other factors such as height of the vegetative hedge, leaf area, number and density of grapevines;

- finally, another important factor is related to operation cost and logistics of current systems. These two aspects lead to collect aerial information only on véraison stage, instead of flowering and maturation stages.

Specification and development of an information collection system of the entire vegetative hedge of the vineyard and all trellis of vines were based on the above-mentioned issues. So, the system was specified to be coupled to a $4 \times 4$ quad-bike or to any agricultural tractor, and can be operated while performing other activities in the vineyard.

\subsection{HW architecture}

The hardware architecture of the system is presented in Fig. 1. The proposed solution comprises the following sensors:

- Inertial sensors (IMU): responsible for providing the position of the camera relative to the vineyard rows;

- GPS/DGPS: responsible for providing the exact position (with high resolution $-0.5 \mathrm{~m}$ ) of the mobile unit and thus to georeference images and maps;

- Cameras: this includes two cameras in visible range and another two in infrared range;

- LIDAR: to acquire depth information, meaning, distance from cameras to the vines.

\section{Inertial sensors - IMU}

The inertial unit is based on the MTi-G from Xsens (www . xsens. com/). This unit has a real time embedded DSP. The

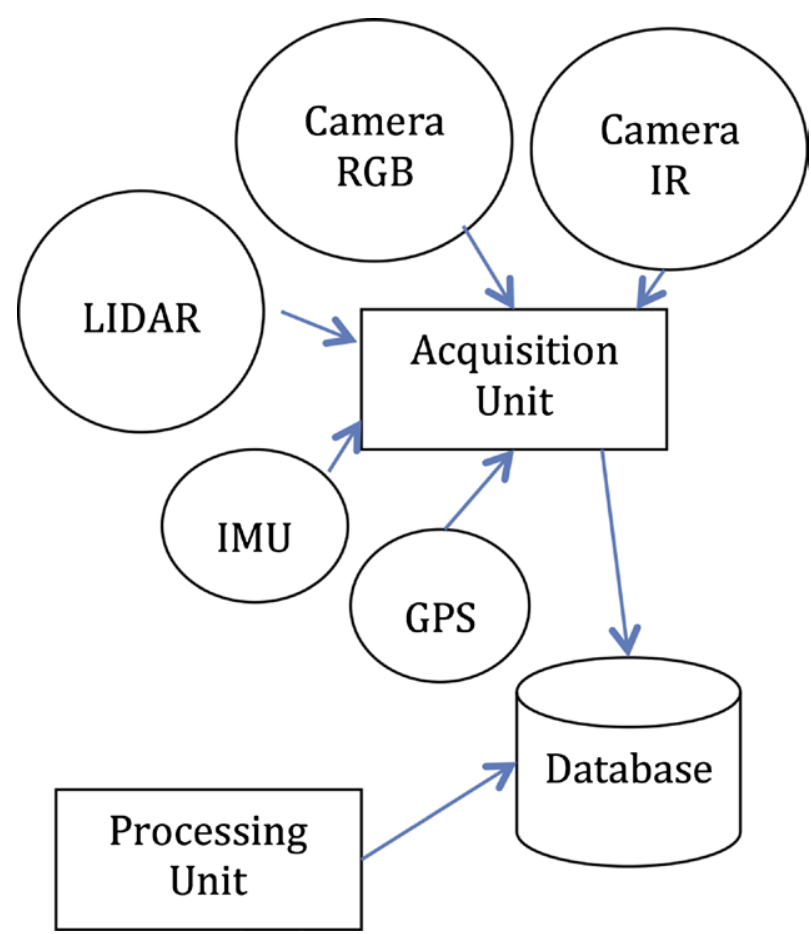

Figure 1. HW architecture.

MTi-G uses miniature MEMS rate gyroscopes and accelerometers. The orientation and position of the MTi-G are estimated using an extended Kalman filter (XKF-6G) of 6 DOF and GPS. The Kalman filter is divided in a prediction step and a correction step. In the prediction step, inertial sensors are integrated over time to estimate position and orientation. In the correction step the drift is corrected using GPS receiver data and the static pressure sensor signal (barometer).

\section{GPS}

The GPS is the EVK 6PPP unit from Ublox (u-blox. com/). Precise Point Positioning (PPP) is a Global Navigation Satellite System (GNSS) positioning method to calculate very precise positions up to few centimeter level using a single (GNSS) receiver in a dynamic and global reference framework like International Terrestrial Frame (ITRF). The EVK 6PPP position accuracy is GPS: $2.5 \mathrm{~m}$; SBAS: $2.0 \mathrm{~m} ;$ SBAS + PPP: <1 m (2D, R50); SBAS + PPP: <2 m (3D, R50).

\section{LIDAR}

The LIDAR system is a low cost unit - UTM-30LX-EW - from Hokuyo-automatic (www.hokuyo-aut.jp/). The UTM-30LX-EW acquires 40 lines/s with a $0.25^{\circ}$ angular resolution. It uses a laser source $(\lambda=905 \mathrm{~nm})$ to scan a $270^{\circ}$ semicircular field. The sensor measures up to three echoes of reflection for each step (direction). Distance and intensity values of every echo are obtained and processed. The system uses two LIDAR, one for each side of the trellis.

\section{Cameras and lenses}

The system uses two cameras UI-3250-C-HQ from IDS (en.ids-imaging.com/). One camera acquires 
images in the visible spectrum, while the other acquires images in the infrared band. In order to obtain IR images, the IR filter was removed from the camera. Combining both cameras a multispectral system was accomplished. Each camera has a resolution of $1600 \times 1200$ pixels ( 19 Mpixels) at a frame rate of $60 \mathrm{fps}$. Also, each one has an HR (high resolution) lens with a focal distance of $3.5 \mathrm{~mm}$. These lenses have high angular angle but with low distortion. The system uses two sets of cameras, one for each side of the trellis.

\section{Processing unit}

The processing unit has an architecture based on desktop PC with Windows 7 Pro. The unit has an i7 processor with 16GB of memory; one disk (for data base) SSD of 2 TB.

\subsection{SW architecture}

To achieve the system goals, the methodological approach included the development of the following algorithms/ modules (Fig. 2):

- modules for HW control and raw data acquisition;

- module for data filtering from raw data to georeferenced data;

- module for post-processing;

- module for classification.

The first block of Fig. 2 controls all data acquisition procedures for LIDAR, IMU, GPS and Cameras. The first stage consisted in the development of procedures for extracting information from inertial sensors and GPS. Then, development continued with tools for image acquisition. This block acquires and saves data at maximum temporal

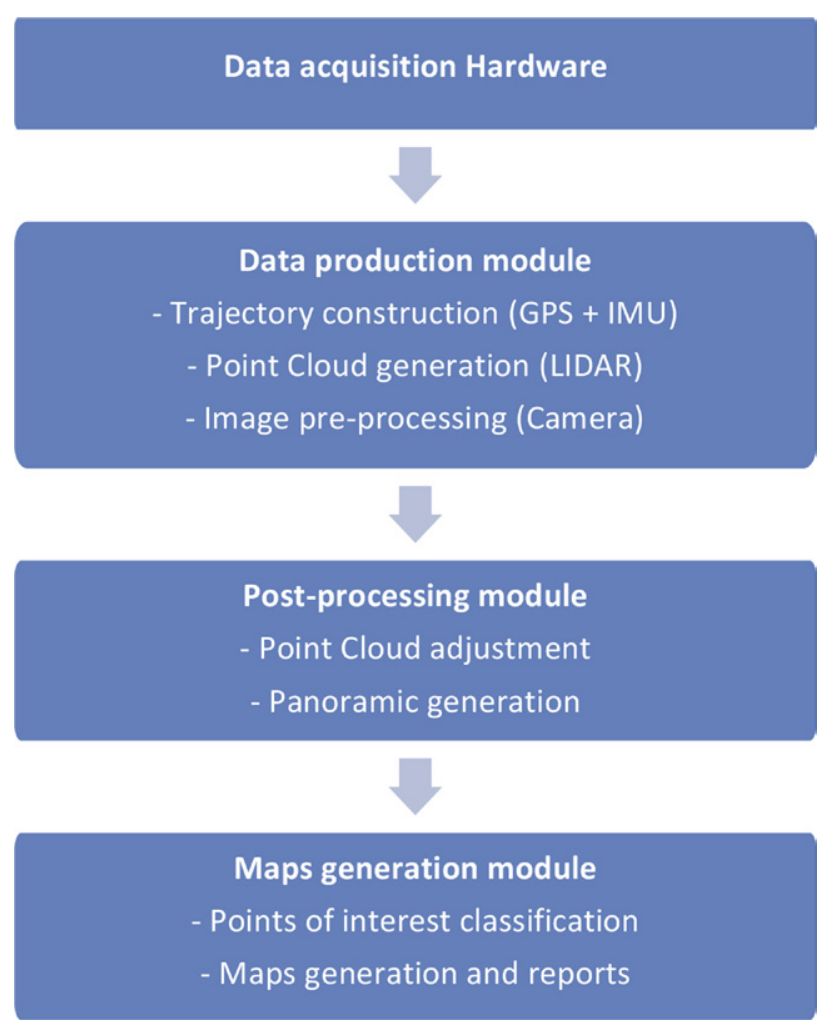

Figure 2. SW architecture. resolution of the system: 40 acquisitions per second. All data are stored for further processing and analysis.

The phase devoted to sensorial fusion (block 2) led to a georeferenced network of "vineyard" and to a projective matrix that allocates all images in the same reference. It uses raw information (IMU data plus raw GPS data) to correct GPS data and to generate the point cloud (obtained by LIDAR) corrected in terms of position (GPS) and in attitude (IMU). Each of the acquired images (RGB and IR) is pre-processed to eliminate noise generated by movement of the cameras and also to establish the reference to the corresponding line of LIDAR. This leads to point cloud and image, which is corrected in terms of the path made by the mobile vehicle.

In the next block (post-processing module) the panoramic image of the height of the vegetative hedge is generated. To do this, initially, incoherent lines of images and point clouds are discharged, using Kalman filters. After this, the mosaic image is constructed using the geo-referenced tags and image registration algorithms. At this point, each portion of the next image fits with the previous image mosaic.

The architecture proceeded with development of algorithms for extracting information that is relevant to create vigour maps. Inputs for this map generation module are the panoramic image and all georeferenced images that prompted it. Through an automatic analysis of images, the system creates standard georeferenced maps: NDVI - Normalized Difference Vegetation Index; PCD - Plant Cell Density; PVR - Photosynthetic Vigour ratio; PPR Plant Pigment Ratio. Besides these indicators, the following items are obtained: recognition of leaf area; height of the vegetative hedge; identification of grapevines, allowing for the determination of the ratio between leaf area $\left(\mathrm{m}^{2} / \mathrm{ha}\right)$ and grape production $(\mathrm{kg} / \mathrm{ha})$; leaf area index.

\section{Results}

The specific goal of the project was the development of a mobile unit, which performs simultaneous image acquisition (in the visible and near infrared), from the left and right trellis, for further processing, analysis and visualization. In this way, $100 \%$ of the vineyard area of a field is acquired, creating a panoramic view of the trellis, with high resolution (in the order of $2 \mathrm{~cm}$ ) as it goes along vine rows.

In order to evaluate results, the prototype was coupled to a quad bike (Fig. 3). Data collection tests were performed in three very different types of terrains and vineyards in Portugal, one in Minho region (Vinho Verde appellation), one in Douro region (Port and Douro appellations) and another in Beiras region (Bairrada appellation).

Figure 4 presents a sequence of three images acquired by the RGB camera and Fig. 5 a panoramic image created using a sequence of these images plus 50 images and the sensor fusion: IMU, GPS and LIDAR.

In the map generation module (graphical user interface in Fig. 6) the operator has access to high-level information (high degree of abstraction): the predefined georeferenced maps. He can also go down in the level of abstraction to more detailed information (sequence of acquired images of the vineyards). In this way, a multiresolution analysis and display is available, as can be seen in Fig. 7. 


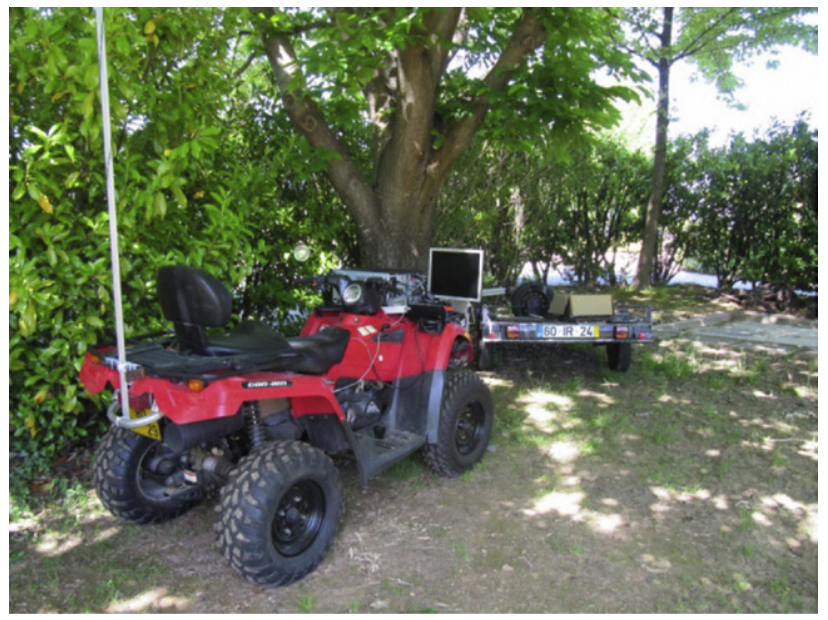

Figure 3. Prototype coupled to a $4 \times 4$ moto.
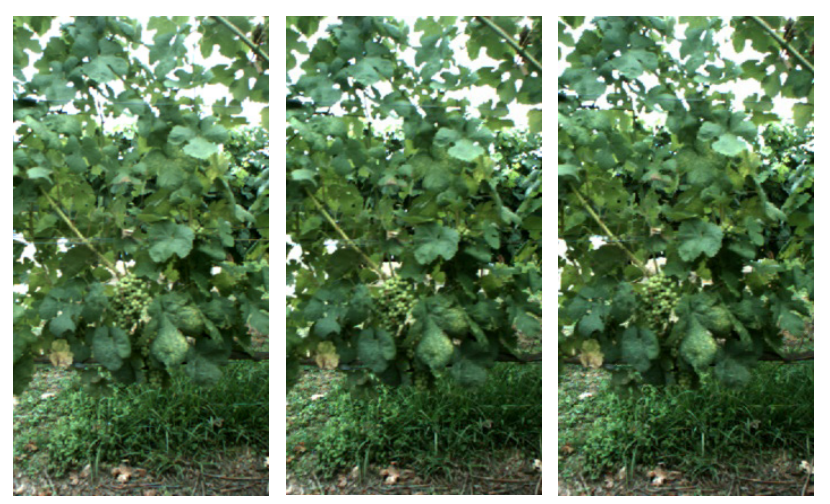

Figure 4. Sequence of 3 acquired images.

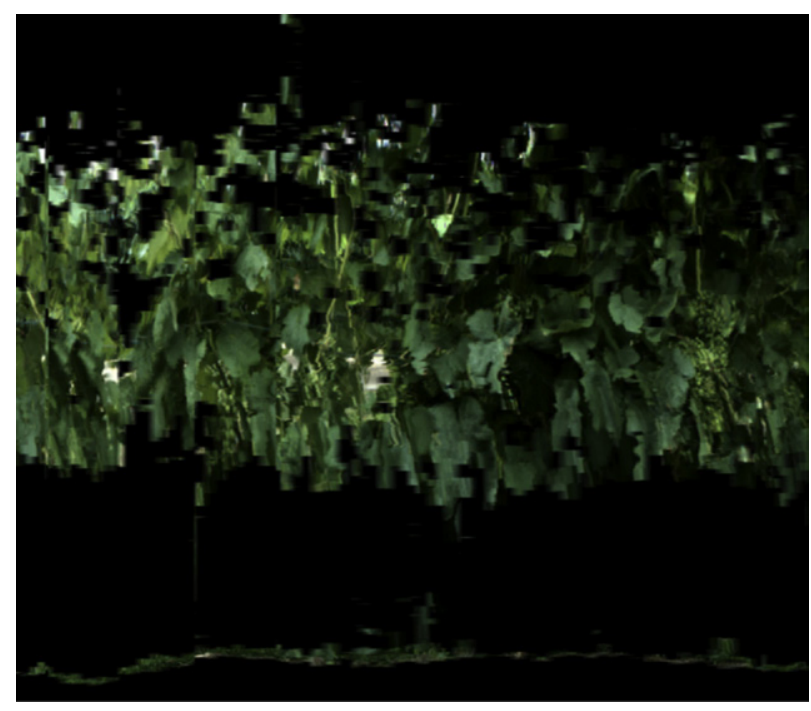

Figure 5. Panoramic image (bottom).

At the intermediate level view (panoramic image) the operator can browse different trellises of vines (Fig. 8).

The panoramic image (Figs. 5 and 8) can be displayed in IR or RGB bands. The data band appearing on the top of the panoramic image (Fig. 8) corresponds to the selected

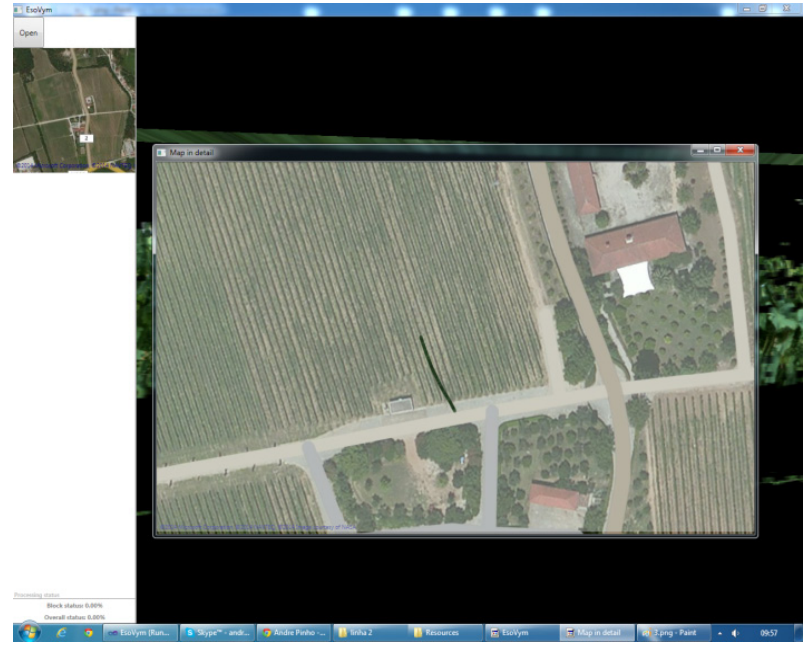

Figure 6. GUI interface.

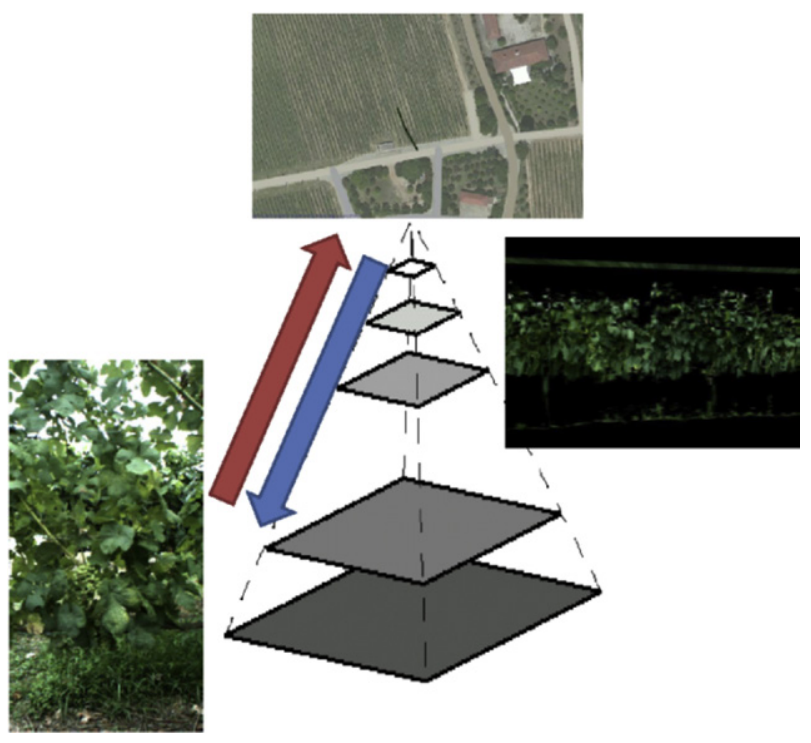

Figure 7. Multiresolution visualization approach.

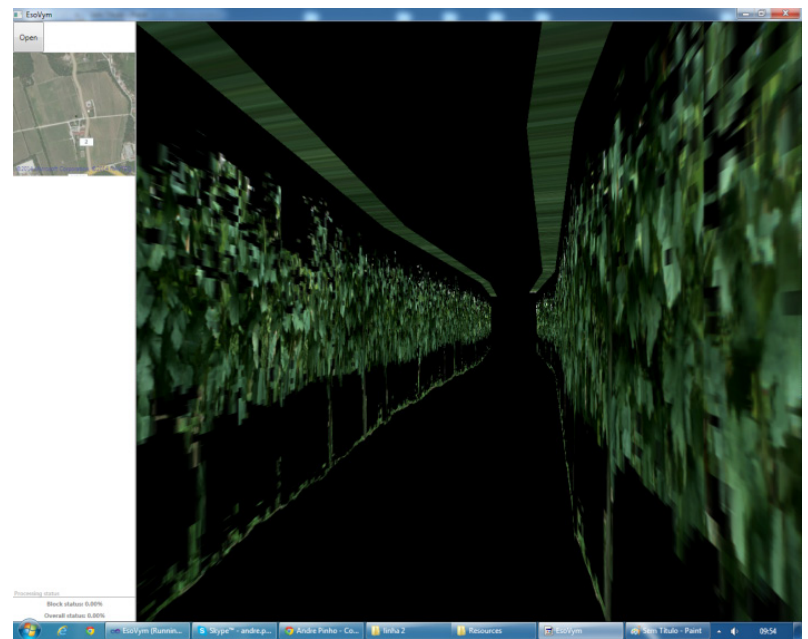

Figure 8. Navigation interface between trellises. 


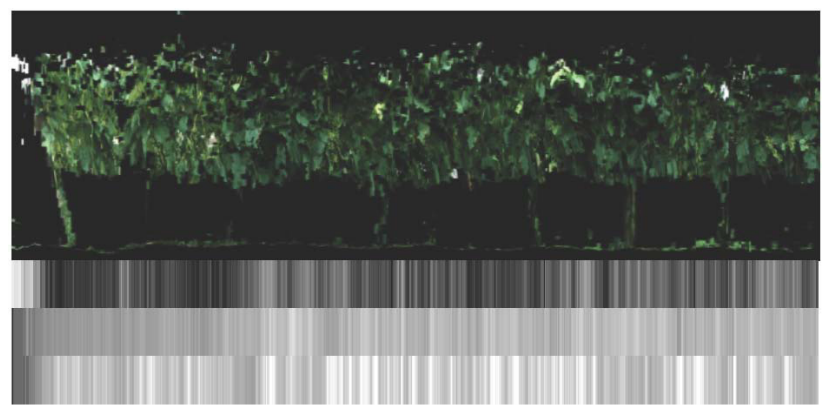

Figure 9. Panoramic image and respective PVR, PPR, and the leaf surface indicators.

information, i.e. the integration of the column in the visible (RGB); or the information in the IR band, or the quantified indicators such as PVR.

As an example, Fig. 9 shows the panoramic image and the respective PVR, PPR, and the leaf surface index indicator bands.

\section{Conclusion}

The proposed system consists of an innovative solution able to generate georeferenced maps of an entire vineyard's canopy surface, based on computer vision techniques, with high resolution, in the order of $\mathrm{cm}^{2}$. An important advantage lies on its ease operation and low cost, which allows use in different stages of production. Also, in terms of function, the system offers the possibility to obtain, remotely, vital data about the state of vineyard production.
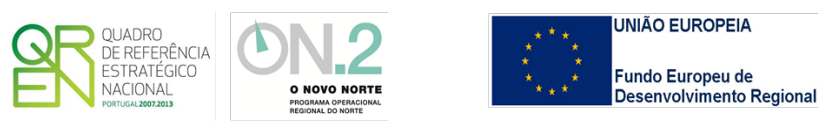

\section{References}

[1] S. Pires, Detecção Remota para aplicações em Viticultura de Precisão no Alentejo. Revista da Associação Portuguesa de Enologia, N.os 51/52 (2008)

[2] D. Lamb, A. Hall, J. Louis, Airbone remoting sensing of vines canopy variability and productivity. Australian Grapegrower \& Winemaker. Annual Technical (2001)

[3] T. Proffit, R. Bramley, D. Lamb, E. Winter, Precision Viticulture. A new era in vineyard management and wine production. Winetitles, Adelaide (2006)

[4] R.G.V. Bramley, Measuring within vineyard variability in yield and quality attributes. Vineyard monitoring and management beyond. Wagga (2000)

[5] R.G.V. Bramley, D.W. Lamb, Making sense of vineyard variability in Australia. Occasional report $\mathrm{n}^{\circ} 14$. Fertilizer and lime research centre, Massey University, Palmerston Nort (2001)

[6] R.G.V. Bramley, D.W. Lamb, Making sense of vineyard variability in Australia. Precision Viticulture, edited by R. Ortega, A. Esser. Proceedings of an international symposium held as part of the IX Congreso Latinoamericano de Viticultura e Enologia, Chile. Centro de Agricultura de Precisión (CAPUC), Pontificia Universidad Católica de Chile (2003)

[7] R.G.V. Bramley, R.P. Hamilton, Understanding variability in winegrape production systems. Australian Journal of Grape and Wine Research. 10: 32-45 (2004)

[8] A. Hall, D.W. Lamb, B. Holzapfel, J. Louis, Optical remote sensing applications in viticulture - a review. Australian Journal of Grape and Wine Research, 8: 36-47 (2002)

[9] R, Ortega, A. Esser, Viticultura de Precisión: Fundamentos, aplicaciones y oportunidades en Chile. Pontificia Universidad Católica de Chile.; 1-10 (2002)

[10] P. Sereno, Viticultura de precisão: Utilização da deteção remota no estudo da variabilidade espacial do vigor, produção e qualidade, castas "SYRAH" E "TOURIGA FRANCA" Universidade Técnica de Lisboa, Master thesis, Lisboa (2009)

[11] C. Pereira, Inspecção visual em aplicações de viticultura de precisão, Universidade de Trás-os Montes e Alto Douro, Master thesis, vila real (2009) 